\title{
UCRL-PROC-231689
}

LAWRENCE LIVERMORE N A T IO N A L LABORATORY

\section{Optimal Design of a Tunable Thomson-Scattering Based Gamma-Ray Source}

D. J. Gibson, S. G. Anderson, S. M. Betts, F. V. Hartemann, I. Jovanovic, D. P. McNabb, M. J. Messerly, J. A. Pruet, M. Y. Shverdin, C. W. Siders, A. M. Tremaine, C. P. J. Barty

June 12, 2007

Particle Accelerator Conference ' 07 Albuquerque, NM, United States June 24, 2007 through June 29, 2007 
This document was prepared as an account of work sponsored by an agency of the United States Government. Neither the United States Government nor the University of California nor any of their employees, makes any warranty, express or implied, or assumes any legal liability or responsibility for the accuracy, completeness, or usefulness of any information, apparatus, product, or process disclosed, or represents that its use would not infringe privately owned rights. Reference herein to any specific commercial product, process, or service by trade name, trademark, manufacturer, or otherwise, does not necessarily constitute or imply its endorsement, recommendation, or favoring by the United States Government or the University of California. The views and opinions of authors expressed herein do not necessarily state or reflect those of the United States Government or the University of California, and shall not be used for advertising or product endorsement purposes. 


\title{
OPTIMAL DESIGN OF A TUNABLE THOMSON-SCATTERING BASED GAMMA-RAY SOURCE*
}

\author{
D. J. Gibson ${ }^{\dagger}$, S. G. Anderson, S. M. Betts, F. V. Hartemann, I. Jovanovic, D. P. McNabb, \\ M. J. Messerly, J .A. Pruet, M. Y. Shverdin, C. W. Siders, A. M. Tremaine, and C. P. J. Barty \\ Lawrence Livermore National Laboratory, Livermore, CA 94550, USA
}

\section{Abstract}

Thomson-Scattering based systems offer a path to highbrightness high-energy $(>1 \mathrm{MeV}) \mathrm{x}$-ray and $\gamma$-ray sources due to their favorable scaling with electron energy. LLNL is currently engaged in an effort to optimize such a device, dubbed the "Thomson-Radiated Extreme X-Ray" (T-REX) source, targeting up to $680 \mathrm{keV}$ photon energy. Such a system requires precise design of the interaction between a high-intensity laser pulse and a high-brightness electron beam. Presented here are the optimal design parameters for such an interaction, including factors such as the collision angle, focal spot size, optimal bunch charge, and laser energy. These parameters were chosen based on extensive modelling using PARMELA and in-house, wellbenchmarked scattering simulation codes.

\section{INTRODUCTION}

Compton- or Thomson-scattering systems, in which incoming high-intensity laser photons scatter off a relativistic electron beam, are doppler-upshifted, and emerge as highenergy $\mathrm{x}$-ray or $\gamma$-ray photons, have shown promise as a new class of light source, with applications ranging from atomic to nuclear to particle physics. Operating in the 10sof $-\mathrm{keV}$ to few-MeV photon energy range, these source can surpass the brightness of synchrotrons by several orders of magnitude due to their straight-forward energy scaling[1]. LLNL has previously demonstrated[2] a bright Thomsonscattering source operating between 30 and $120 \mathrm{keV}$. This system was used, among other things, to carefully benchmark Compton-scattering simulation codes[3].

Optimization of photon brightness is key to many applications. Here we study the optimal design of the interaction geometry for a Thomson-scattering source. Important parameters to define include the charge of the electron bunch, the energy in the scattering laser field, the laser pulse duration, the angle of the interaction collision, and the spot size of the laser and electron beams at the focus.

\section{INTERACTION GEOMETRY}

The first decision to be made in the design of a Thomsonscattering source is what interaction angle to use between

\footnotetext{
${ }^{*}$ This work was performed under the auspices of the U.S. Department of Energy by University of California Lawrence Livermore National Laboratory under contract No. W-7405-Eng-48.

† gibson23@1lnl.gov
}

the beams. The scattered x-ray energy $\hbar \omega_{x}$ is given by

$$
\hbar \omega_{x}=2 \gamma^{2} \frac{1-\cos \theta_{i n c}}{1+\gamma^{2} \theta_{o b s}^{2}} \hbar \omega_{l}
$$

where $\gamma=\sqrt{1-v^{2} / c^{2}}$ is the Lorentz factor of the electron, $\theta_{i n c}$ and $\theta_{\text {obs }}$ are the angles between the electron momentum vector and the incident photon momentum vector and observed x-ray direction, respectively, and $\hbar \omega_{l}$ is the energy of the scattering photon. This value is maximized for an interaction at $\theta_{i n c}=180^{\circ}$.

For these $180^{\circ}$ collisions, the duration of the scattered $\mathrm{x}$-ray pulse depends almost entirely on the electron pulse length and is, to first order, independent of the laser duration. For applications where $\mathrm{x}$-ray pulse lengths on the few ps time scale are acceptable, the $180^{\circ}$ geometry offers advantages over other angles. One is that the photons see the entire length of the the electron bunch $(\approx 3 \mathrm{~mm})$, not just the width $(\approx 20 \mu \mathrm{m})$, so the column density of scatterers is higher, thus maximizing the flux. Also, the system becomes less sensitive to timing jitter; a slip in the relative timing of the two beams causes the interaction point to move longitudinally, but does not change the scaattering column densities. A more detailed discussion of this issue, with simulations, is found in [2].

\section{PULSE LENGTH}

In the $180^{\circ}$ geometry, the duration of the laser pulse is determined not by considerations of the x-ray pulse length but by the energy spread in the bunch it can produce. Shorter laser pulses require more bandwidth which means a bigger spread in $\omega_{l}$. This maps directly into a greater spread in $\omega_{x}$, which causes brightness to suffer. Also, longer pulses allow more photons to be put into the interaction region without raising the electric field strengths up to nonlinear intensities, as discussed below. The only significant limit on the pulse length comes from the Rayleigh range of the laser and beta function of the electron beam, which define the interaction length. If the laser pulse is longer than this length, the photons at the ends of the distribution won't see the tightly focussed electrons and will contribute only minimally to the scattering. Thus, a pulse length on the order of $10 \mathrm{ps}$ is a nominal upper bound on the laser length.

\section{BUNCH CHARGE}

The optimal bunch charge for the electron beam depends on a balancing act between the number of scatterers avail- 
able and the electron beam emittance, which correlates directly with $\mathrm{x}$-ray beam brightness. On one hand, we want to maximize the number of electrons at the interaction point to increase the total number of scatterers available at the interaction point to generate x-rays. On the other hand, high charge density at the photocathode when the beam is created results in strong space-charge forces which cause the emittance of the beam to rise. This emittance, when it reaches the interaction point, translates into a spread in electron direction, which due to the beam-like nature of the scattering process, results in a larger divergence $\mathrm{x}$-ray beam. Because of the angular dependance of the scattered photon energy seen in Eq. 1, this spread also results in an increase in the width of generated x-ray spectrum. For experiments involving narrow linewidths, this means fewer photons at the right energy will be available.

The electron bunch was modeled at various charges (see Fig. 1 for values) using PARMELA[4], assuming a uniform cylinder of charge at the photocathode. For each charge $q$, the initial electron spot size and length at the photocathode was scaled as $q^{1 / 3}$ to maintain the charge density and minimize the changes that would need to be made to the linac parameters. Because the initial spot size varies as a function of the charge, and the thermal emittance contribution is linearly dependent on the initial beam radius, it is important to include thermal emittance effects in these calculations. Simulations were run with $\varepsilon_{t h, n}=0.9 r_{b} \mathrm{~mm} \mathrm{mrad} / \mathrm{mm}$, where $r_{b}$ is the beam hard-edge radius[5]. The thermal emittance was added in PARMELA by generating random transverse momenta for the initial particles. No energy spread was applied to the electrons. The solenoid strength was varied for each of the runs to optimize the emittance at the entrance to the first accelerator section, but the resulting field strength always optimized to the same value. The electrons were then accerlated to $112 \mathrm{MeV}$ in 5 shortened SLAC-type accelerating sections. Between the first and second accelerator sections, a quadrupole triplet, made up of three $10 \mathrm{~cm}$ quads with $5 \mathrm{~cm}$ gaps between them, was used to "collimate" the electron beam. The values used for the fields of each quad was set by using TRACE-3D[6] to model an electron focus at the end of the second section. It was observed in the runs that the beam size was well behaved (slowly shrinking) along the remaining 4 sections of the accelerator without any further focusing, so no additional quads were used in the beam transport.

At the end of the accelerator a quadrupole triplet, with effective lengths of 10,20 , and $10 \mathrm{~cm}$ with $5 \mathrm{~cm}$ gaps between, was used to focus the electron beam. Again, TRACE-3D was used to find the ideal field strengths for the magnets, and the focal length was also allowed to vary to get a bunch with the target spot size. A focused rms spot size of $\sigma_{x}=10 \mu \mathrm{m}$ was used for the $1 \mathrm{nC}$ bunch and $\sigma_{x}$ was adjusted for each bunch based on its emittance $\varepsilon_{x}$ to create a divergence at the focus that matched that of a $1.1 \mathrm{~mm}$ mrad bunch focusing to $10 \mu \mathrm{m}$ (i.e. $\varepsilon_{x} / \sigma_{x}$ is constant). To produce the PARMELA output for the Compton-scattering code, 50,000 particles were run through the linac and to the focus, and the 6-dimensional coordinates of each of them was recorded.

The Compton-scattering process was simulated using custom software[7], which takes the electron distribution at focus from PARMELA and propagates them through the laser field, calculating the scattered intensity. Here, the laser used to simulate $\mathrm{x}$-ray production was assumed to be a 355-nm, 1-J, 5-ps-FWHM, fourier-transform-limited pulse with a spot size adjusted to match the electron spot size in each case $\left(w_{0}=\sigma_{x}\right)$. The code assumes ballistic electron trajectories and the Thomson scattering limit, and therefore doesn't include any nonlinear effects, so the results may not be realistic for the smaller spot sizes with higher $a_{0}^{2}$. It is assumed the system is apertured to allow only a $1 \mathrm{mrad}$ half-angle cone to pass (to limit the bandwidth). The code integrated the spectrum over all time and over this cone. The end result, shown in Fig. 1, is that if thermal emittance effects are included, the optimal bunch charge occurs in a broad peak around $1 \mathrm{nC}$.

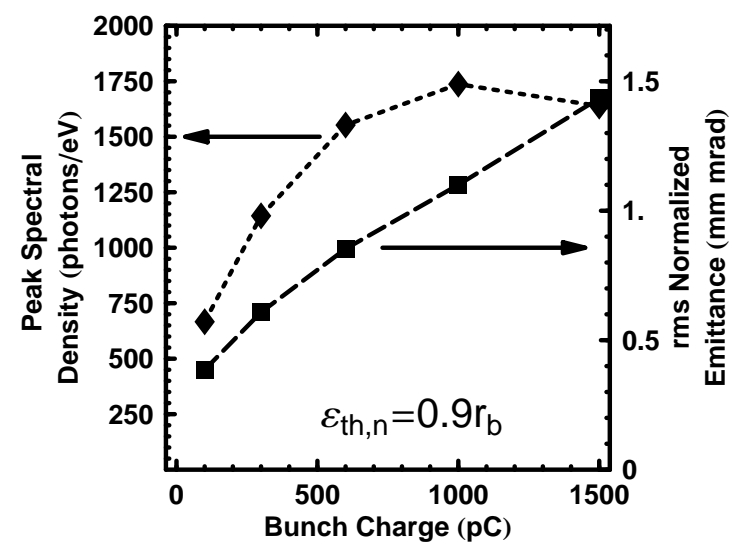

Figure 1: Emittance and peak spectral density in $1 \mathrm{mrad}$ half-angle cone for $\varepsilon_{t h, n}=0.9 r_{b} \mathrm{~mm} \mathrm{mrad} / \mathrm{mm}$ as a function of electron bunch charge.

\section{LASER ENERGY}

It is important to keep the photon density low enough to avoid nonlinear effects in the scattering process. If the laser field strength is too high, the motion of the electrons in the field will become relativistic, causing the scattered photon spectrum to downshift, broaden, and in severe cases to generate harmonics[1]. All these effects will have a negative impact on the brightness of the x-ray source as they result in fewer of the scattered photons being at the targeted source energy. Choosing to fix the square of the normalized vector potential of the laser, which is directly proportional to the photon density, at a value of $a_{0}^{2}=10^{-3}$ keeps the field strength low enough to avoid any appreciable broadening due to nonlinear effects. This requirement also ties the total laser energy directly to the focal spot size. 


\section{FOCAL SPOT SIZE}

Since the photon density is limited by nonlinear consideratons, and the laser and electron focal spot sizes have to be matched to each other to maximize the overlap, the choice of a focal spot size is driven by electron beam considerations.

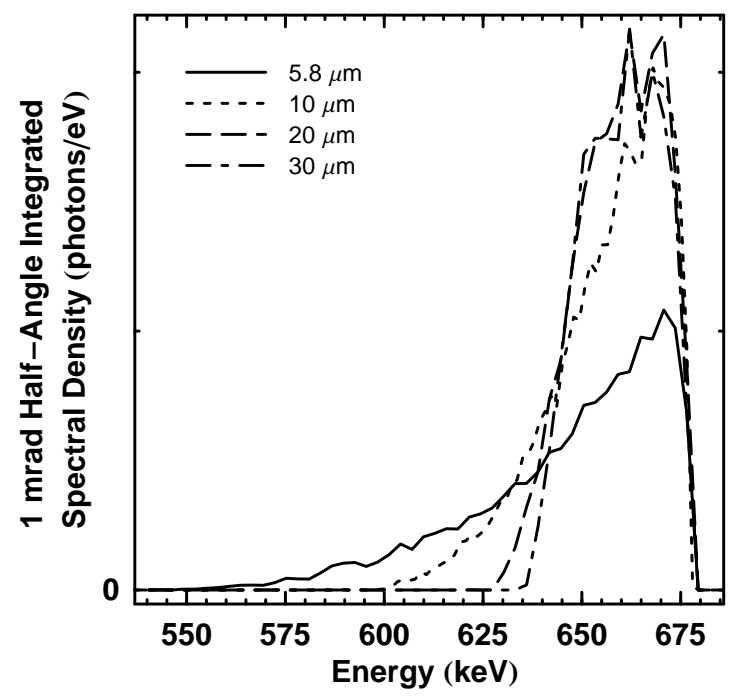

Figure 2: 1 mrad half-angle cone integrated spectra plotted for various focal spot sizes. A $20 \mu \mathrm{m}$ spot produces the brightest spectrum with the least laser energy.

A $1 \mathrm{nC}$ bunch, propagating through the accelerator described above, was focused to a variety of rms spot sizes from $\sigma_{x}=5.8 \mu \mathrm{m}$ to $\sigma_{x}=30 \mu \mathrm{m}$. Fig. 2 shows the resultant spectra integrated over a $1 \mathrm{mrad}$ half-angle divergence cone for each of the spot sizes using the same method described above for the bunch charge study. In this case, the scattering laser beam energy was varied with the spot size to maintain the constant photon density given by the $a_{0}^{2}=10^{-3}$ requirement. For the small spot sizes, the convergence angle of the beam to get to the small spot causes significant broadening of the spectrum due to the greater range of scattering angles. As the spot size shrinks, the spectrum narrows until it reaches the point where the angular spread is dominated not by the correlated transverse momentum from the focusing element, but by the uncorrelated transverse momentum resulting from the emittance. As seen in Fig. 2, as the spot size goes from $20 \mu \mathrm{m}$ to 30 $\mu \mathrm{m}$ the spectrum does not get any narrower or taller. Since the spot is now more than twice the area, however, the laser energy also needs to more than double to maintain the same energy density. Thus, $\sigma_{x}=20 \mu \mathrm{m}$ is the optimal spot size for our system, and this correlates to a laser beam energy of $360 \mathrm{~mJ}$ at $351 \mathrm{~nm}$ and $10 \mathrm{ps}$ pulse length.

\section{CONCLUSIONS}

Detailed modeling of a Thomson-scattering based $\mathrm{x}$ ray $/ \gamma$-ray source, from electron bunch generation at the photocathode, through the entire 5-section linear accelerator and to the focus, and through the photon scattering process using scattering codes we've developed and benchmarked, have yielded an optimal design space for constructing a new system. Trying to optimize the bunch to have a minimal bandwidth, maximal flux, and not being concerned with the pulse duration, we have found the proper design point to be a $360 \mathrm{~mJ}, 10 \mathrm{ps}, 20 \mu \mathrm{m}$ laser interacting with a $1 \mathrm{nC}, 10 \mathrm{ps}, 20 \mu \mathrm{m}$ electron beam. A system built to these parameters is nearing the end of its construction at LLNL.

\section{References}

[1] F. V. Hartemann, W. J. Brown, D. J. Gibson, S. G. Anderson, A. M. Tremaine, P. T. Springer, A. J. Wootton, E. P. Hartouni, and C. P. J. Barty, Phys. Rev. Spec. Top. - Accel. and Beams 8, 100702 (2005).

[2] D. J. Gibson, S. G. Anderson, C. P. J. Barty, S. M. Betts, R. Booth, W. J. Brown, J. K. Crane, R. R. Cross, D. N. Fittinghoff, A. M. Tremaine, A. J. Wooten, E. P. Hartouni, P. T. Springer, and J. B. Rosenzweig, Phys. Plasmas 11, 2857-2864 (2004).

[3] W. J. Brown, S. Anderson, C. P. J. Barty, S. Betts, R. Booth, J. K. Crane, R. R. Cross, D. N. Fittinghoff, D. J. Gibson, F. Hartemann, E. P. Hartouni, J. Kuba, G. P. L. Sage, D. R. Slaughter, A. Tremaine, A. J.Wootton, and P. Springer, Phys. Rev. Spec. Topics - Accel. Beams 7, 060702 (2004).

[4] L. Young, and J. Billen, Parmela, Tech. Rep. LA-UR96-1835, Los Alamos National Laboratory (1996).

[5] J. F. Schmerge, J. E. Clendenin, D. H. Dowell, and S. M. Gierman, Rf gun photo-emission model for metal cathodes including time-dependent emission, Tech. Rep. SLAC-PUB-11700, Stanford Linear Accelerator Center (2006).

[6] K. R. Crandall, and D. P. Rusthoi, Trace-3d documentation, Tech. Rep. LA-UR-97-886, Los Alamos National Laboratory (1997).

[7] W. J. Brown, and F. V. Hartemann, Phys. Rev. Spec. Topics - Accel. Beams 7, 060703 (2004). 